\title{
Spatial Representations of Hippocampal CA1 Neurons Are Modulated by Behavioral Context in a Hippocampus-Dependent Memory Task
}

\author{
Amy L. Griffin, Howard Eichenbaum, and Michael E. Hasselmo \\ Center for Memory and Brain, Boston University, Boston, Massachusetts 02215
}

\begin{abstract}
Although it is well known that hippocampal neurons code spatial information, it is less clear how these spatial representations are influenced by memory demands, especially in hippocampus-dependent tasks. Recently, our laboratory has demonstrated that hippocampal spatial representations are influenced by mnemonic factors in a T-maze continuous alternation task. Another unique experimental approach that might reveal the ways in which task-related factors impact hippocampal spatial representations is to compare firing patterns between events that require distinct episodic memory processes. Therefore, we recorded from CA1 single neurons during a discrete trial delayed-nonmatch-to-place task that allowed within-trial comparison between an encoding (sample) phase and a retrieval (choice) phase. A large subset of neurons that fired on the central stem of the maze showed dramatic selectivity for either the sample or choice phase of the trial. However, surprisingly, there were fewer neurons that showed differential firing rates between left- and rightbound trajectories. Our results suggest that trial-phase-selective coding is common in tasks that require rapid alternation between encoding and retrieval processes.
\end{abstract}

Key words: hippocampus; context; delayed-nonmatch-to-place; episodic memory; encoding; retrieval

\section{Introduction}

Spatial navigation in rodents has been widely used as a model system for understanding the computations performed by the hippocampus during episodic memory. Place cells, neurons in the hippocampus that fire selectively when the rat is in a specific location in the environment, are perhaps the most striking evidence that the hippocampus participates in forming spatial representations (O'Keefe and Dostrovsky, 1971; O'Keefe and Nadel, 1978; Muller and Kubie, 1987). However, a critical issue is whether these spatial firing patterns reflect a mapping of space or the encoding and retrieval of places in which memorable events occur. One approach that may distinguish between spatial mapping versus episodic memory views is to record from hippocampal neurons during tasks in which memory can be directly assessed, for example, during tasks in which traversals through the same place are parts of different overall experiences. Several experiments have demonstrated that CA1 spatial representations are clearly modulated by experience in tasks in which animals traverse the same locations as parts of different overall trajecto-

Received Sept. 18, 2006; revised Jan. 29, 2007; accepted Jan. 30, 2007.

This work was supported by National Institutes of Health (NIH) Grants 5 F32 MH070184-03 (A.L.G.) and DA16454 [as part of the NIH-National Science Foundation (NSF) program for Collaborative Research in Computational Neuroscience]: MH60013 (M.E.H.); NSF/Science of Learning Centers/Social, Behavioral, and Economic Sciences 0354378 (M.E.H., H.E.); and MH51570 and MH071702 (H.E., M.E.H.). We thank the following people for their technical assistance: Tucker Larsen, Prateek Aggarwal, Eric Zilli, and Michael Economo. We also thank Inah Lee for his thoughtful comments on a previous version of this manuscript.

Correspondence should be addressed to Amy L. Griffin, Center for Memory and Brain, 2 Cummington Street, Boston University, Boston, MA 02215. E-mail: amygriff@bu.edu.

DOI:10.1523/JNEUROSCI.4083-06.2007

Copyright $\odot 2007$ Society for Neuroscience $\quad$ 0270-6474/07/272416-08\$15.00/0 ries, with each study reporting that a significant proportion of cells show trajectory-dependent firing (Frank et al., 2000; Wood et al., 2000; Ferbinteanu and Shapiro, 2003; Bower et al., 2005). However, these studies have not determined whether the differential firing patterns are related to the encoding and retrieval of memories as described in a recent computational model (Hasselmo and Eichenbaum, 2005) or instead are means by which the hippocampus disambiguates similar sequences of movement through a familiar environment. Here we address this issue by using a discrete-trial delayed-nonmatch-to-place (DNMP) paradigm, which requires continuous switching between cue-based encoding and memory-dependent retrieval phases of each trial. Unlike in the continuous alternation task (E. Wood, personal communication) performance on the DNMP task is sensitive to hippocampal damage (Shaw and Aggleton, 1993; Hampson et al., 1999; Dudchenko et al., 2000). Thus, one might expect a prominent representation of memory-related aspects of the task similar to that seen in other hippocampus-dependent tasks (Wible et al., 1986; Ferbinteanu and Shapiro, 2003).

We recorded from multiple CA1 single neurons during the hippocampus-dependent discrete-trial DNMP task. Many neurons showed a striking selectivity for the task phase, suggesting that these cells were sensitive to the memory demands of the task, with some neurons participating in encoding on the sample phase and some neurons participating in retrieval on the choice phase.

\section{Materials and Methods}

Animals and surgery. Four male, Long-Evans rats (weighing 450-500 g) were individually housed and maintained on a $12 \mathrm{~h}$ light/dark cycle with 
ad libitum access to food and water. During periods of behavioral testing, rats were given four to five pellets of food per day to keep them at $80-90 \%$ of their free-feeding body weight. For implantation of electrodes, rats were anesthetized with isoflurane, the skull surface was exposed, seven anchor screws and one ground screw (located at the lambda skull suture) were affixed to the skull, and a $2 \mathrm{~mm}$ hole was drilled for the six-tetrode microdrive. The microdrive contained six tetrodes (composed of four $12.7-\mu \mathrm{m}$-diameter nichrome wires, $150-300 \mathrm{k} \Omega$ at $1 \mathrm{kHz}$ in gold solution), a reference electrode, and a local field potential recording electrode. The dura was removed from the microdrive target area, and the drive was lowered onto the brain surface. The screws and microdrive were attached to the skull with dental acrylic, and each tetrode was immediately advanced $160 \mu \mathrm{m}$. The rats were allowed to recover for 1 week before behavioral testing and recording began. All animal procedures and surgery were in accordance with National Institutes of Health and Boston University Animal Care and Use Committee guidelines.

Recording protocol. After recovery from surgery, the reference electrode was lowered until it reached the area immediately dorsal to the CA1 pyramidal cell layer. Another electrode was lowered into the area of the hippocampal fissure to record local field potentials. The six tetrodes were then advanced gradually over the course of 5-7 d and positioned in the CA1 layer by monitoring units and local field potentials. Neural signals were preamplified by unity-gain operational amplifiers located on the head stage of the rat. The signals were then amplified $(2000-10,000 \times)$ and bandpass filtered $(0.3-6 \mathrm{kHz}$; Neuralynx, Tucson, AZ). Whenever a unit crossed the channel amplitude threshold (set by the experimenter), all four channels of the tetrode were digitized at $32 \mathrm{kHz}$ and stored (Neuralynx). The position data were recorded from a camera mounted above the maze that captured $(30 \mathrm{~Hz})$ luminance emitted from an array of light-emitting diodes located on the rat head stage.

Training protocol. The maze was a T-maze $(116 \times 107 \mathrm{~cm}$ long, $10 \mathrm{~cm}$ wide) that was modified with return arms ( $112 \mathrm{~cm}$ long, $10 \mathrm{~cm}$ wide) to allow the rat to return to the bottom of the stem of the maze after visiting a reward zone. Each reward zone contained a rimmed plastic disc that was baited with chocolate sprinkles. The plastic disc and chocolate sprinkles were both dark in color, so the rat could not see the reward before reaching the reward zone. The holding box was a rotating pedestal located $28 \mathrm{~cm}$ away from the base of the maze. The rat traveled between the maze and the pedestal via a removable ramp $(40 \mathrm{~cm}$ long, $11 \mathrm{~cm}$ wide). The testing room was illuminated with a $25 \mathrm{~W}$ incandescent bulb located above the maze. The testing room walls contained large visual cues that remained unchanged throughout the experiment. During behavioral testing and pretraining, white noise was played through speakers to mask any outside noise that may have distracted the rat and added confounds to the interpretation of the neural data.

Before surgery, each rat was handled and acclimated to the testing environment. After recovery from surgery, rats were given 20 - 40 forcedchoice trials identical to the sample phase of the DNMP trials described below every day until the first recording session. Figure $1 \mathrm{~A}$ shows a schematic of the DNMP task. Each trial consisted of two stem traversals (laps), both starting when the rat began running up the stem of the maze and ending when the rat stopped at a reward zone. Immediately before each trial, one of the two reward arms (perpendicular to the maze stem) was blocked with a large opaque barrier. The experimenter placed the ramp between the maze and pedestal, allowing the rat to traverse from the pedestal to the maze. The sample phase of the trial began when the rat exited the ramp, consisted of the rat running up the stem and into the unblocked reward arm, and ended when the rat reached the reward zone. After consuming the reward, the rat then returned to the base of the maze stem via the return arm and waited there for a delay period of $10 \mathrm{~s}$. During this time, another opaque barrier prevented the rat from proceeding up the stem of the maze. The choice phase then began when the barrier was removed, and the rat was allowed to proceed up the stem of the maze and make a free choice between a left and right turn response. Visits to the reward zone opposite to that visited on the sample phase were rewarded with another chocolate sprinkle. After the choice phase, the rat ran back down the return arm and back to the pedestal via the ramp. The ramp was then removed, and the rat waited on the pedestal for an intertrial interval (ITI) of 10-20 s while the experimenter prepared for the next trial. The
A.

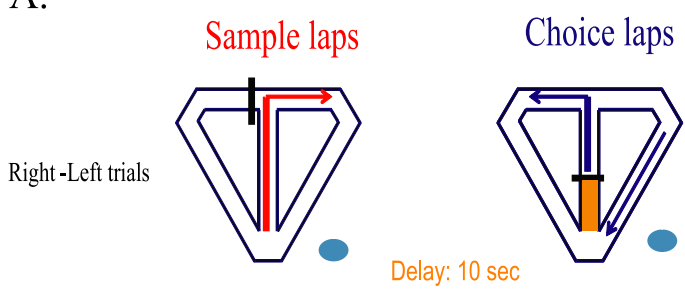

ITI: $10-20 \mathrm{sec}$

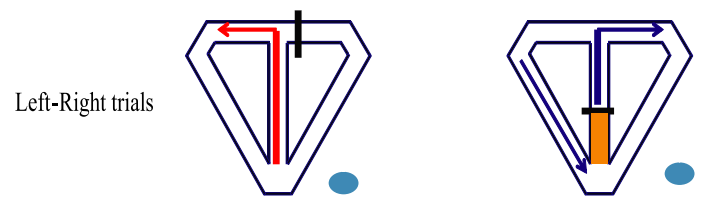

B.

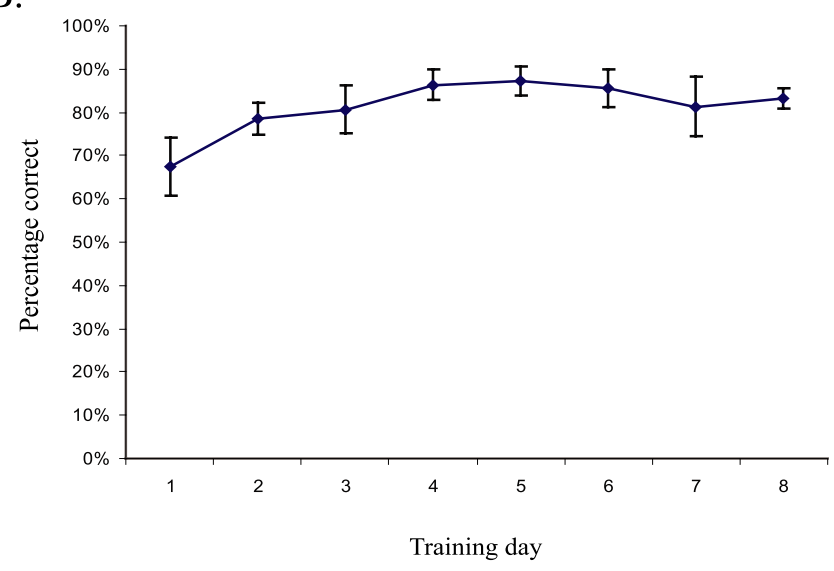

Figure 1. The DNMP task. $A$, Schematic diagram of the DNMP task. For each trial, the sample phase began when the rat entered the maze from the holding pedestal (blue oval) via a moveable ramp and ended when the rat entered either the right or left reward zone, depending on which reward arm was available. The rat then returned to the base of the stem (orange shaded region) and was held there for a $10 \mathrm{~s}$ delay period. The choice phase began when the delay barrier was removed. For the choice phase, the rat was allowed to choose between a left or right lap, with reward provided only for traversals to the reward arm opposite to that visited on the sample lap. The rat then returned to the holding platform for an intertrial interval of $10-20 \mathrm{~s}$, during which the experimenter set up for the next trial. Rats were given $20-40$ trials per day for $8-10 \mathrm{~d}$. The trials were given in pseudorandom order, with approximately equal numbers of right-left and left-right trials given in a daily session. $\boldsymbol{B}$, Average percentage of correct trials for the four rats across the first $8 \mathrm{~d}$ of training. Rats performed well above chance even on the first day of the task. Spatial correlates of hippocampal neurons were analyzed only for sessions in which the percentage of correct trials was $80 \%$ or greater (error bars indicate SEM).

rats were given 20-40 trials each day, presented in a pseudorandom sequence (no more than three of the same trial type in a row, approximately equal numbers of left and right trials).

Histology. At the end of the experiment, a small marking lesion was made by passing $10 \mu \mathrm{A}$ through one wire of each tetrode. Rats were then perfused transcardially with formalin (4\%), and the head was soaked in formalin for 2-3 d. The tetrodes were then raised up out of the brain and the brain was removed from the skull. Frozen sections $(40 \mu \mathrm{m})$ were mounted on slides using a cryostat and stained using Neutral red (a Nissl stain). Tetrode tracks were reconstructed using both tetrode depth profiles and by examination of the sections under a microscope. Only data obtained from tetrodes verified histologically to be located in the CA1 pyramidal layer were included in the experiment.

Data analysis. Units were isolated using standard cluster cutting techniques and custom software. Only clusters containing $>100$ spikes and satisfying an established isolation quality threshold (Lee et al., 2006) were 
included in the analysis. Pyramidal cells were distinguished from interneurons based on previous work (Ranck, 1973). Briefly, units were considered to be pyramidal cells based on waveform shape, a mean overall firing rate $<5 \mathrm{~Hz}$, and a bursting pattern revealed by the interspike interval (ISI) histogram.

A custom Matlab program (MathWorks, Natick, MA) calculated instantaneous velocity for each spike and filtered out spikes that occurred while the animal was stationary or moving at a low velocity $(<10 \mathrm{~cm} / \mathrm{s})$. This filtering method ensured that any spike included in the analysis was not reflective of irrelevant movements (i.e., leaning over the maze wall) or of sharp wave activity that occurred during grooming or eating.

Event timestamps were manually assigned to the beginning and end of each lap using a custom Matlab program. Then, each spike in a given cluster was assigned to one of four lap types: left sample, right sample, left choice, and right choice.

For each cluster with a statistically significant $(p<0.01)$ information score (Skaggs et al., 1996), the return arm, stem, and reward arms of the maze were divided into 56 consecutive $5 \mathrm{~cm}$ bins. For each lap type, the bin firing rate was calculated by dividing the number of spikes the cell fired while the rat occupied the bin by the bin occupancy. For analysis purposes, the sample phase began when the rat entered the maze stem from the waiting platform and ended when the rat entered the reward zone. The choice phase began when the barrier was removed after the delay period and ended when the rat entered the reward zone. The delay zone was defined as the area covering the seven bins at the base of the stem in which the rat was held between the sample and choice phases. Firing rate distributions consisting of 56 bins were then created for each of the four lap types. The linearized firing rate distributions for each lap type were smoothed using a Gaussian filter (full-width half-maximum of 4 bins). A firing field was defined as described previously (Lee et al., 2006): four or more contiguous bins with a firing rate of at least $10 \%$ of the maximum bin firing rate. Therefore, the field boundaries were defined as bins in which the firing rate dropped below $10 \%$ of the maximum firing rate. The field size was then calculated by subtracting the bin containing the field boundary closest to the reward zone from the bin containing the field boundary closest to the beginning of the stem and multiplying by the bin size $(5 \mathrm{~cm})$. To identify stem-firing units, the center of mass (COM) was calculated as described previously (Mehta et al., 1997, 2000; Lee et al., 2004, 2006). A field was included in the analysis if, for any of the 4 lap types, the COM was located on the stem of the maze and the mean firing rate for the stem exceeded $0.5 \mathrm{~Hz}$.

To compare stem firing rates between the four types of trajectories, the mean firing rate for the 13 bins covering the area of the stem outside of the delay zone was calculated for each of the four lap types. Thus, this analysis included only the firing of neurons when the rat was in the area of the stem beyond the delay barrier and excluded firing that occurred while the rat was in the delay zone and the area of the stem immediately adjacent to the delay barrier location. To compare firing rates between right and left laps on the stem of the maze, a discrimination index ( $\left.\mathrm{DI}_{\text {turn }}\right)$ was calculated using the following formula:

$$
\mathrm{DI}_{\text {turn }}=\frac{\mathrm{FR}_{\mathrm{r}}-\mathrm{FR}_{1}}{\mathrm{FR}_{\mathrm{r}}+\mathrm{FR}_{\mathrm{l}}},
$$

where $\mathrm{FR}_{\mathrm{r}}$ is the mean stem firing rate on right turn laps, and $\mathrm{FR}_{1}$ is the mean stem firing rate on the left turn laps $( \pm 1.0$ is perfect discrimination, 0.0 is no discrimination). Similarly, sample and choice phases were compared using the following formula:

$$
\mathrm{DI}_{\text {phase }}=\frac{\mathrm{FR}_{\mathrm{s}}-\mathrm{FR}_{\mathrm{c}}}{\mathrm{FR}_{\mathrm{s}}+\mathrm{FR}_{\mathrm{c}}},
$$

where $\mathrm{FR}_{\mathrm{S}}$ is the mean stem firing rate on the sample phase, and $\mathrm{FR}_{\mathrm{C}}$ is the mean stem firing rate on the choice phase. For statistical comparison of firing rates between sample and choice laps and between left and right laps, the maze stem was divided into four equal segments consisting of three bins each. Then, a 2 (task phase) $\times 2$ (turn direction) $\times 4$ (stem segment) ANOVA was performed on each unit. Units were considered to be trial-phase selective if there was a main effect of trial phase or a phase $X$ turn direction or phase $\times$ stem segment interaction. Similarly, units were considered to be turn-direction selective if there was a main effect of turn direction or if there was a turn direction $\times$ phase or turn direction $\times$ stem segment interaction. Note that individual units could be selective for both turn direction and trial phase. To reduce the possibility that firing rate differences between lap types were attributable to the delay barrier cutting the firing field in half, a second 2 (task phase $) \times 2$ (turn direction $) \times 4$ (stem segment) ANOVA was performed on units whose field centers (COM) were located outside of the delay zone (i.e., within the 13 bins used to calculate mean stem firing rate as described above). Additionally, for each stem-firing unit, we calculated a $\mathrm{DI}_{\text {phase }}$ value comparing firing rates between the sample and choice laps for times when the rat was in the reward arms, in which sensory cues and behavior were identical for sample and choice laps.

To verify that lap-type selectivity was not a result of velocity differences

\section{Table 1. Number of units recorded during stable performance across rats and} sessions

\begin{tabular}{llcc}
\hline Rat & $\begin{array}{l}\text { Number of sessions at } \\
\text { 80\% correct or higher }\end{array}$ & Number of units & Number of stem-firing units \\
\hline 1 & 3 & 33 & 3 \\
2 & 4 & 32 & 10 \\
3 & 7 & 110 & 22 \\
4 & 6 & 165 & 42 \\
\hline
\end{tabular}

A

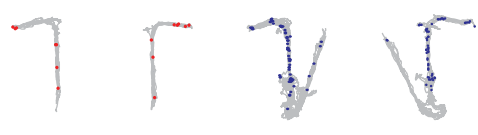

Mean firing rate $=0.5 \mathrm{~Hz}$

B

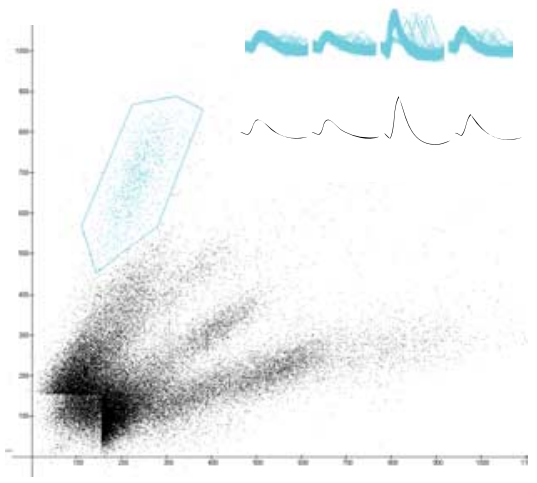

Figure 2. Firing characteristics of a choice-selective neuron. $A$, Unit firing locations for sample (red dots) and choice (blue dots) phases of the task superimposed on the position data (gray traces). $\boldsymbol{B}$, Distribution of waveform heights in two-dimensional cluster cutting space, with each dot representing the relative spike heights recorded on two channels of a tetrode. Cluster boundaries of the unit in $\boldsymbol{A}$ are outlined in green. Inset, All waveforms (top) and average waveform (bottom). $\boldsymbol{C}$, Interspike interval histogram, autocorrelogram, and time versus maximum height plot for the unit in $A$. Notice the bursting pattern in the ISI histogram (top), the peak at $t=0$ in the autocorrelogram (middle), and the stability across the recording session (bottom). 
Table 2. Categorization of units based on firing properties and field locations

\begin{tabular}{ll}
\hline Firing location & Number of units \\
\hline Delay zone & 45 \\
Stem & 87 \\
Return arms & 35 \\
Reward arms & 17 \\
Pedestal/ramp & 58 \\
Sparse firing & 87 \\
Interneurons & 11 \\
\hline
\end{tabular}

Sparse firing neurons emitted $<100$ spikes in a session. Pyramidal cells were distinguished from interneurons based on the shape of the interspike interval histogram, waveform shape, and firing rate (see Materials and Methods).

Choice-selective neurons

Sample-selective neurons
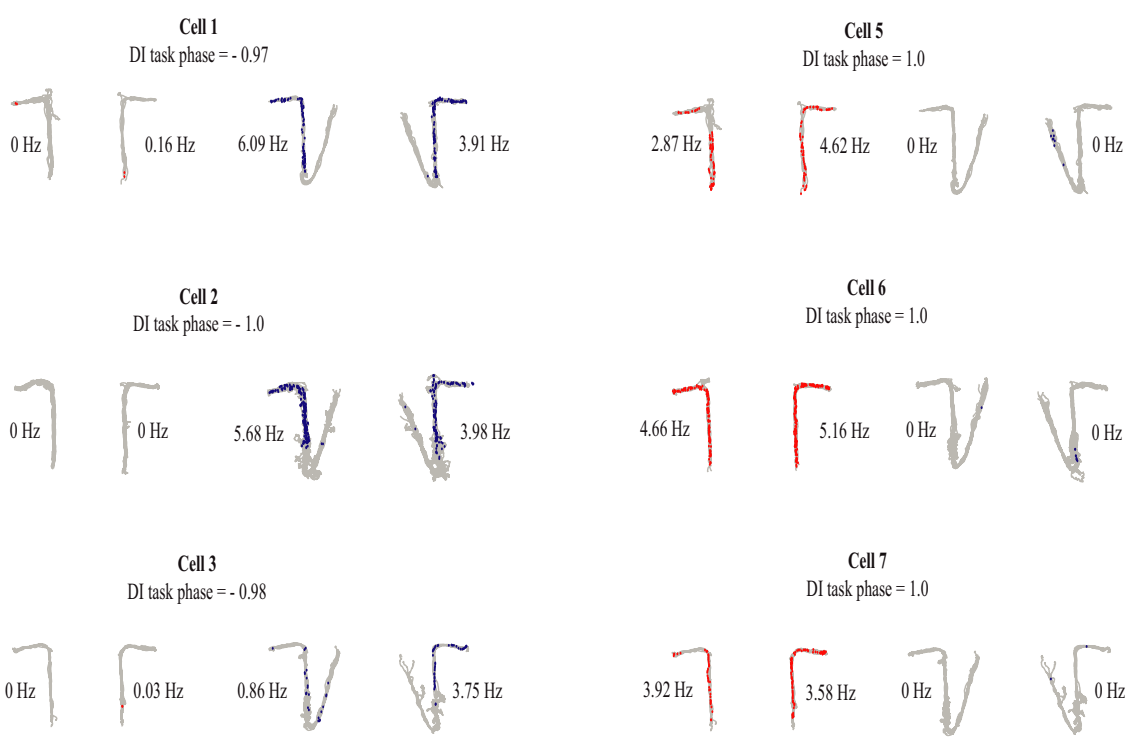

Cell 4
DI task phase $=-0.96$

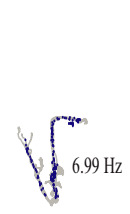

$0.29 \mathrm{~Hz} \quad \int_{0.05 \mathrm{~Hz}}^{-}$
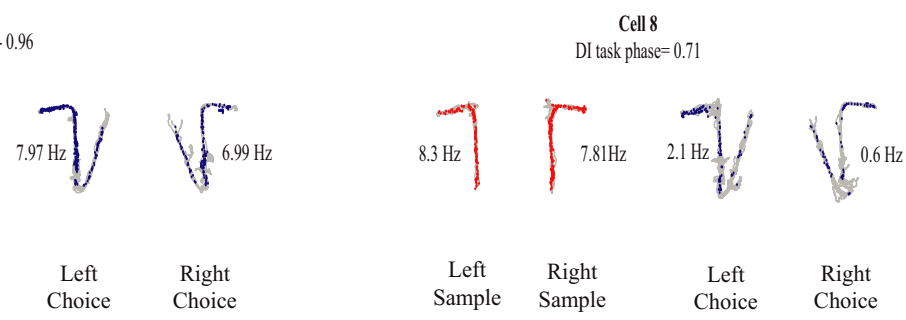

\section{Results}

tions). Individual fields were considered to show forward shifting if there was a significant correlation between $\triangle C O M$ and trial.

We used independently movable tetrodes to record multiple single neurons from CA1 in four rats while they performed a DNMP task. Each daily session consisted of 20-40 trials (with each trial including a sample and a choice phase). Only units recorded during sessions in which the rat performed at $80 \%$ correct or better were included in the analysis (Table 1). Rats readily acquired the task, with the majority of errors being on the first few trials of the first training day and performance being well above chance thereafter (Fig. $1 B$ ). Of the 340 units that met the criterion for adequate cluster isolation, 329 were determined to be pyramidal cells based on criteria from previous work (Ranck, 1973) (see Materials and methods). Figure 2 shows firing characteristics and isolation quality of a representative pyramidal cell that fired on the maze stem during the DNMP task. Note the bursting pattern in the ISI histogram, the single peak at $t=0$ in the autocorrelogram, and the stability in the waveform amplitude across the session (Fig. 2C).

\section{Stem-firing neurons showed trial- phase-specific firing during the DNMP task}

The remainder of the analysis focuses on the cells that were active as the animals traversed the central stem of the maze (Table 2). There were 87 units with firing fields on the stem of the maze. However, there was one session in which the rat ran significantly faster on sample laps than on choice laps. Excluding the 10 units that were recorded during this session, 77 units were used in the subsequent analyses. There were no velocity differences between right-turn and left-turn laps in any session and no systematic differences in path deviation between any of the types of laps (data not shown). For each unit, we compared the stem firing rate between four lap types: left sample, right sample, left choice, and right choice. As seen in Figure 3 and Table 4, most of the fields

between lap types, $t$ tests were performed that compared the average running velocity on the stem between sample and choice phases and between left and right laps. If any significant differences were found between lap types in velocity in any session, the units recorded during the session were excluded from the analysis. Similarly, we also examined the path deviation (distribution of horizontal variation in the position coordinates) between the phases for each session to verify that firing rate differences were not a result of differences in the spatial trajectory taken on each phase.

To investigate the time course of the fir-ing field location, the difference $(\triangle \mathrm{COM})$ between the COM for the entire set of trials $\left(\mathrm{COM}_{\text {session }}\right)$ and for each trial $\left(\mathrm{COM}_{\text {trial }}\right)$ was calculated for all units that had a COM on the maze stem, a firing rate of at least $1 \mathrm{~Hz}$, and that were recorded in a session with at least 12 of each lap type. Note that each unit could have up to four fields (one for each of the four lap-type firing rate distribu- extended the entire length of the trajectory, spanning over $80 \%$ of the total path for sample laps and $\sim 50 \%$ of the total path for choice laps.

Figure 3 shows the notable finding that many of the units fired selectively depending on the task phase (i.e., fired robustly on sample laps and minimally on choice laps, or fired robustly on choice laps and minimally on sample laps). To quantify this observation, we calculated a sample versus choice discrimination index $\left(\mathrm{DI}_{\text {phase }}\right)$, ranging from -1.0 to +1.0 , which reflected the degree of differential firing between sample and choice phases, with values close to -1.0 indicating choice-phase selectivity, values close to 1.0 indicating sample-phase selectivity, and values close to 0 indicating low task-phase selectivity. The selectivity for 
trial phase was prominent across the population. Figure 4 shows the distribution of $\mathrm{DI}_{\text {phase }}$ and $\mathrm{DI}_{\text {turn }}$ values across all stem-firing neurons. A large number of units showed a strong preference for either sample or choice phases as indicated by the number of values near \pm 1.0 in $A$. In contrast, although a substantial portion of units showed turn direction specificity, this proportion was relatively low compared with the number of units showing preference for task phase as indicated by the clustering of $\mathrm{DI}_{\text {turn }}$ values around 0 in $B$.

For statistical confirmation of these findings, the stem of the maze was divided into four equal segments and a 2 (trial phase) $\times$ 2 (turn direction) $\times 4$ (stem segment) ANOVA was performed. As shown in Table 3, this analysis revealed that 38 of 77 units showed a main effect of trial phase, 11 units showed a trial phase $\times$ turn direction interaction, and five units showed a trial phase $\times$ stem segment interaction. In total, 54 of 77 units (70\%) showed either main effects or interactions that indicated trialphase selectivity, firing selectively on either sample or choice laps. In addition, a substantial, albeit smaller proportion of cells clearly differentiated left turn and right turn trials. Of the 77 units analyzed, 14 (18\%) showed a significant main effect of turn direction or a turn direction $\times$ trial phase interaction. Finally, there were 20 units $(26 \%)$ that did not show any trial phase or turn direction effects and therefore could be considered to have traditional place fields on the stem of the maze.

It is possible that trial-phase selectivity, especially in the case of sample-selective units, was attributable to fields being bisected by the barrier used to confine the rats to the base of the stem during the delay period as suggested by previous findings (Muller and Kubie, 1987). Therefore, we performed an additional analysis excluding all units with a field center located in the bottom half of the stem, and we only considered the activity of these cells during the period when the animal was in the top half of the stem. This exclusion left 44 units. As seen in Table 3, 28 of the 44 units (73\%) were trial-phase selective. Because most of the fields that we observed extended throughout the trajectory, it could be argued that even the fields with a COM outside of the delay zone were bisected by the delay barrier. Six of the stem-firing units had field boundaries entirely outside of the delay zone (i.e., in the part of the stem closest to the choice point). All of these units showed significant phase selectivity. Specifically, of these six fields, four showed a significant main effect of phase and had $\mathrm{DI}_{\text {phase }}$ values that exceeded \pm 0.6 (three were choice selective and one was sample selective), one showed a phase $\times$ turn direction $\times$ stem segment interaction and a $\mathrm{DI}_{\text {phase }}$ value of 0.5 (sample selective), and one showed a phase $\times$ stem segment interaction and a $\mathrm{DI}_{\text {phase }}$ value of -0.45 (choice selective). We also calculated $\mathrm{DI}_{\text {phase val- }}$ ues comparing the mean firing rate between sample and choice laps during visits to the reward arms. Importantly, during reward arm traversals, the behavioral and sensory variables were identical between sample and choice laps, because the barriers were not visible to rats during these times. As seen in Figure $4 C$, the distribution of $\mathrm{DI}_{\text {phase }}$ values for the reward arms was similar to the distribution of $\mathrm{DI}_{\text {phase }}$ values for the maze stem, with many units showing preferred firing on either the sample or the choice trial phases.

Because there were so few error trials after rats reached asymptotic performance, it is not possible to use quantitative methods to compare the firing properties on correct and incorrect trials for the current experiment. However, examination of the pattern of activity on individual error trials shows that phase-selective neurons appear to maintain their selectivity on error trials.
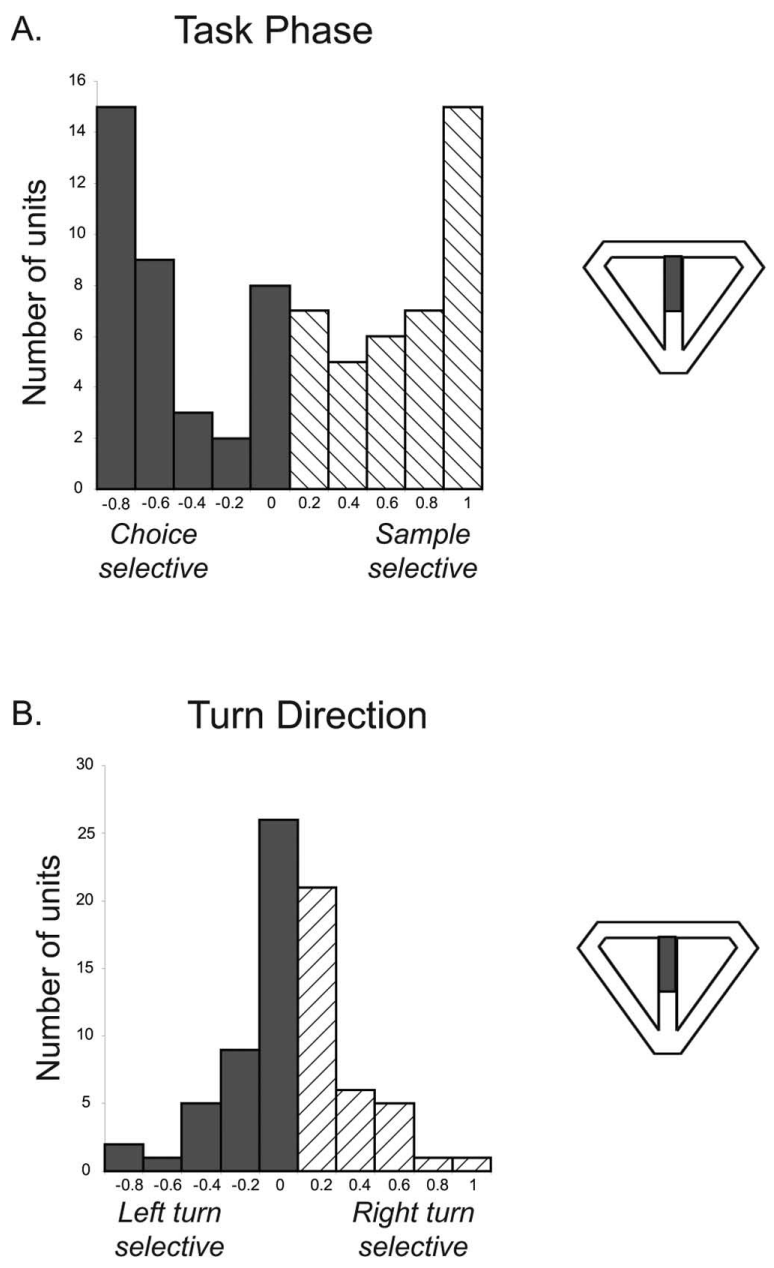

C.
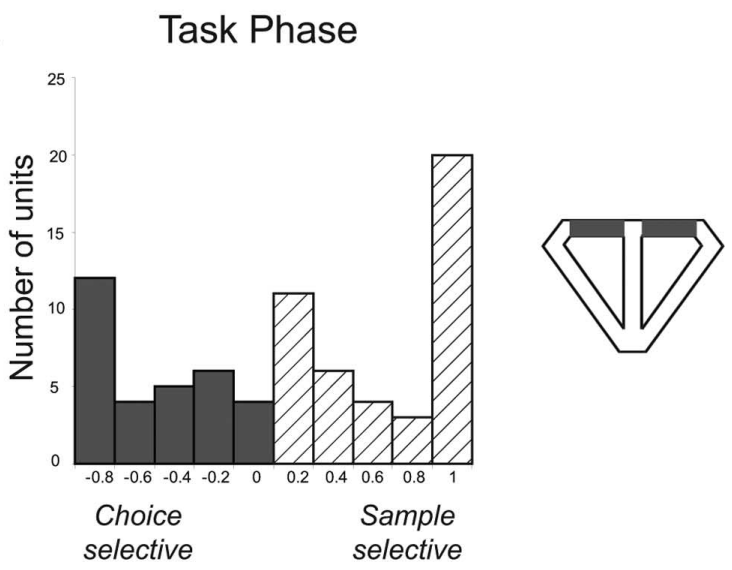

Discrimination Index

Figure 4. Distribution of discrimination indices for all stem-firing neurons. The area of the maze that was used for firing rate comparisons is indicated by the diagram to the right of each plot. $\boldsymbol{A}$, Comparison of firing rates on the maze stem between sample and choice laps ( $\left.\mathrm{DI}_{\text {phase }}\right)$. Cells with a DI $>0$ fired more robustly on sample than on choice laps, and cells with a $\mathrm{DI}<0$ fired more robustly on choice laps than on sample laps. Notice the high proportion of cells with DI values near 1 and -1 and approximately equal numbers of sample-selective and choiceselective units. $\boldsymbol{B}$, Distribution of discrimination indices ( $\mathrm{DI}_{\text {turn }}$ ) for all stem-firing neurons comparing right-turn and left-turn trials. Although a small number of units showed turn selectivity, many neurons were not sensitive to turn direction as indicated by the clustering of DI values around $0 . C$, Distribution of $\mathrm{DI}_{\text {phase }}$ values comparing firing rates between sample and choice laps in the reward zones, in which sensory and behavioral variables did not differ between sample and choice laps. Notice the similarity to the distribution in $\boldsymbol{A}$. 
Table 3. Main effects and interactions of context-sensitive factors from the three-factor ANOVA

\begin{tabular}{|c|c|c|c|c|c|}
\hline Trial phase effects & $\begin{array}{l}\text { Number of units } \\
\text { (percentage of total) }^{b}\end{array}$ & $\begin{array}{l}\text { Number of units excluding } \\
\text { bisected fields (percentage } \\
\text { of total) }\end{array}$ & Turn direction effects & Number of units & $\begin{array}{l}\text { Number of units } \\
\text { excluding bisected } \\
\text { fields }\end{array}$ \\
\hline Trial phase & $38(49.4)$ & $20(45.5)$ & Turn direction & $3(3.9)^{a}$ & $2(4.5)$ \\
\hline Trial phase $\times$ turn direction & $11(14.3)$ & $6(13.6)$ & Trial phase $\times$ turn direction & $11(14.3)$ & $6(13.6)$ \\
\hline \multicolumn{6}{|l|}{ Trial phase $\times$ stem seg- } \\
\hline ment & $5(6.5)$ & $2(4.5)$ & Turn direction $\times$ stem segment & $0(0)$ & $0(0)$ \\
\hline
\end{tabular}

${ }^{a}$ These units showed a main effect of both trial phase and turn direction.

${ }^{b}$ This analysis was performed on 77 units.

'This analysis was performed on 44 units (excluding clusters whose field center was located in the delay zone).

A.

$$
\Gamma
$$<smiles>c1ccccc1</smiles>

Field 1<smiles>CCCCCC</smiles>

Field 2
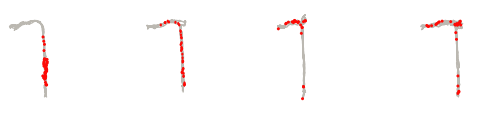

Field 3

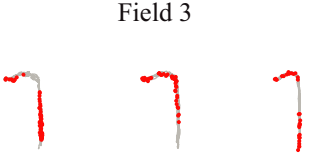

Field 4

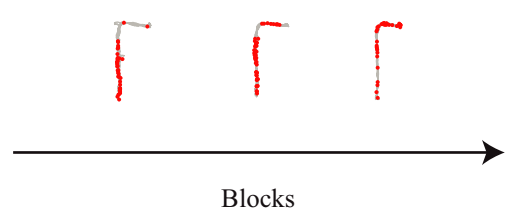

B.

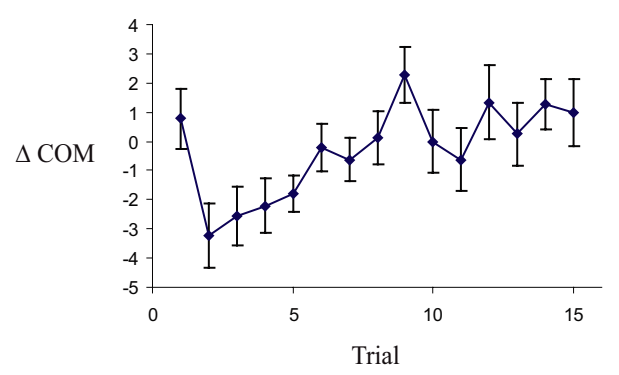

Figure 5. Linear forward shift in the firing fields toward reward zones. $A$, Examples of the time course of the firing field location from four separate firing fields over the first 15 trials of the session (red dots, spike locations; gray traces, position data). The trials are grouped in blocks of three. $B, \Delta C O M$ shift for all 50 firing fields. There was a significant linear relationship between $\Delta C O M$ and $\operatorname{trial}(r=0.64 ; p<0.01)$. Notice that there was also a backward $\Delta$ COM shift over the first two trials of the session $[t$ (paired) $=$ 2.79; $p$ (one-tailed) $=0.004]$.
Time course of trial-phase-specific firing during the training session

Our next step was to assess whether the trial-phase-specific firing fields shifted their spatial representation forward on the maze throughout the training session as was seen in a previous continuous alternation study (Lee et al., 2006). To adequately assess the time course of the firing field shift, we selected firing fields that had a $\mathrm{COM}$ on the maze stem, a firing rate of at least $1 \mathrm{~Hz}$, and that were recorded in a session with at least 12 of each lap type. These restrictions yielded 50 firing fields from 24 units ( 9 fields from left choice firing rate distributions, 16 fields from left sample firing rate distributions, 11 fields from right choice firing rate distributions, and 14 fields from right sample firing rate distributions). Note that some units had firing fields for more than one lap type. Fields were considered to be shifting fields if there was a significant $(p<0.05)$ correlation between $\triangle \mathrm{COM}$ and trial. There were 10 fields that showed a significant forward shift across trials. Figure $5 \mathrm{~A}$ shows examples of fields that showed a forward shift in their firing field throughout the training session.

The mean shift in the $\triangle \mathrm{COM}$ was also calculated for the full population of neurons fulfilling the selection criterion described above. The mean $\triangle \mathrm{COM}$ data are plotted in Figure $5 B$, showing a significant linear shift forward on the maze across trials $(r=0.64 ; p<0.01)$. This forward shift across the population contributes to the relatively large size of firing fields summarized in Table 4. As seen in Figure 5B, the forward shift in firing field was preceded by a backward shift similar to that seen on unidirectional laps study by Lee et al. (2006) and previous tasks without a memory demand (Mehta et al., 1997, 2000). Specifically, there was a significant backward shift in the mean $\triangle \mathrm{COM}$ from trial 1 to trial $2[t$ (paired) $=2.79 ; p$ (onetailed) $=0.004]$. In summary, the fields shifted backward from trial 1 to trial 2 and subsequently showed a gradual forward shift across the remainder of the session. 


\section{Discussion}

In the current study, we used a hippocampus-dependent spatial working memory task to make direct comparisons between hippocampal firing rates during encoding and retrieval. We found that the majority $(70 \%)$ of hippocampal CA1 neurons that had firing fields on the stem of the maze fired preferentially in one of two trial phases: the encoding (sample) phase or the retrieval (choice) phase.

The effect of context on the firing properties of hippocampal neurons has been reported in several spatial working memory studies (Frank et al., 2000; Wood et al., 2000; Ferbinteanu and Shapiro, 2003). We observed a smaller percentage of turnselective units compared with previous investigations, which found turn selectivity ranging from 36 to $70 \%$ of the cells (Lee et al., 2006: 70\%; Wood et al., 2000: 67\%; Ferbinteanu and Shapiro, 2003: 59\%; Bower et al., 2005: 55\% in the "skipped reward" task; Frank et al., 2000: 36\%). However, the percentage of turndirection-selective units that we observed was arguably clearly sufficient for discriminating turn direction on the basis of neural activity. The difference between our results and those of previous studies is likely attributable to the different memory demands required by the two tasks. In contrast to other investigations, our task consisted of trials that included a sample phase, in which it was important to encode the current trajectory and a choice phase, in which it was important to retrieve the previous trajectory. Most of the neurons in our study fired selectively on either the cue-based sample phase of the trial or memory-driven choice phase of the trial. Some studies by other investigators reported varying extents of turn-selective firing on an overlapping segment of two distinct trajectories For example, hippocampal neurons fired similarly between left-bound and right-bound laps in a Y-maze (Lenck-Santini et al., 2001b). Bower et al. (2005) ran a series of experiments showing degrees of differential firing along an overlapping segment of two distinct trajectories depending on the location of the reward sites and the initial training regimen. Together, these findings suggest that the hippocampal trajectory coding scheme can vary dramatically between similar behavioral paradigms depending on the details of the experimental protocol and, in some cases, on the memory requirements of the task. In the hippocampus-dependent DNMP task, there is substantial disambiguation of left and right turn paths as well as coding for discrete spatial locations. However, these representations become secondary, in terms of the proportion of cells involved, to distinguishing between events that require encoding (the sample phase) and events that require retrieval (the choice phase).

Task-related firing patterns resembling those seen in the current study have been observed in other hippocampus-dependent memory tasks. CA1 neurons have been shown to fire preferentially to task phase in both a delayed-match-to-sample (DMS) (Hampson et al., 1993) and a delayed nonmatch-to-sample (DNMS) (Deadwyler et al., 1996) lever-pressing task, to match or nonmatch trials in a odor DNMS task (Wood et al., 1999), and during the sample or choice phase of a DMS runway task (Wible et al., 1986). Together with our results, these findings indicate that, in addition to spatial information and route disambiguation, the hippocampus codes specific features of the testing environment along with salient events occurring within that environment, all of which may be used by the rat to optimally perform a memory-guided task.
The DNMP task used in the current study requires the animal to run along four similar trajectories that differ only in cue dependence (sample vs choice) and goal location (right vs left). On the sample phase, the navigation strategy is entirely cue driven, because the barrier blocking one of the goal arms acts as a cue to enter the opposite goal arm. An alternative explanation for our findings is that sample-selective and choice-selective units were driven by the presence or absence, respectively, of the barrier that was used on the sample phase. It is well known that hippocampal spatial representations can be modified by changes in context (Anderson and Jeffery, 2003; Smith and Mizumori, 2006) and manipulation of environmental cues (Muller and Kubie, 1987; Tanila et al., 1997; Lenck-Santini et al., 2001a; Fyhn et al., 2002; Leutgeb et al., 2005). The earliest suggestion of this kind was O'Keefe and Nadel's (1978) description of "misplace" cells that are driven by the addition or removal of salient objects within a familiar and otherwise stable environment. Muller and Kubie (1987) found that introducing a barrier into a familiar environment caused cells to remap, but only if the barrier was inserted directly into the center of the place field. Because we analyzed only cells that had firing fields on the maze stem, most fields were not bisected by the sample phase barrier. Furthermore, if the barrier used on the sample phase caused remapping, one might expect more turn-selective units especially on the sample phase in which the barrier location differs in a very relevant way between left and right trials. It is still possible that trial-phase selectivity can be attributed to the fact that the delay zone barrier bisected the sample lap fields. To address this issue, we performed a selective analysis on only those units with the field centroid located in the top half of the stem (nearest to the choice point). As seen in Table 3, when units with fields that were likely bisected by the delay barrier were removed, $73 \%$ of units still showed trial-phase selectivity. However, even fields not bisected by the barriers in the current study could have been influenced by the change in visual cues between sample and choice phases. Rivard et al. (2004) showed that fields rotate along with barriers placed adjacent to the field boundaries. Similarly, another study (Gothard et al., 1996b) showed that some fields followed a reward-signaling landmark or a moveable start box, suggesting that external cues can control place cell firing. However, the fields that were influenced by these cues were located in close proximity to either the goal landmark or the start box. In the current study, the phaseselective firing fields included large areas that were a sizeable distance away from the barriers. Furthermore, as shown in Figure $4 C$, trial-phase selectivity occurred even in the reward arms, in which no barriers are visible to the rat and in which the sensory and behavioral factors are identical for sample and choice laps. Therefore, although it is unlikely that the sample and delay barriers caused the phase selectivity, future experiments could explicitly eliminate this potential confound by making the entire sample and choice trajectories identical.

There have been multiple reports of a shift in firing field loca- 
tions over the course of the training session. In most cases, the shift was induced by manipulating local or distal cues in the environment (Gothard et al., 1996a,b; Rosenzweig et al., 2003) or by shifting or adding reward locations (Breese et al., 1989; Kobayashi et al., 1997; Hollup et al., 2001). Mehta et al. (1997, 2000) reported changes in place field shape and location that occurred in the absence of changes to the task or environment. Specifically, as rats made multiple passes through a place field, the fields expanded backward and became negatively skewed. A more recent study showed that firing fields that were selective for one of two trial types in a continuous T-maze alternation task showed a dramatic shift forward on the stem of the maze, toward reward locations (Lee et al., 2006). Here, we also observed a withinsession forward shift in the firing fields. This shift cannot be explained by a shift in the reference frame between lap types as observed by other investigations (Gothard et al., 1996a,b). If this was the case, we would have seen a systematic shift forward in the field from a sample lap to a choice lap and a shift back for the next sample lap. Instead, we observed a gradual forward shift within a specific lap type. This result suggests that the forward shift in spatial representations is not restricted to the continuous alternation task and thus may play a role in episodic encoding rather than simple lap disambiguation.

In conclusion, the DNMP task requires an overall coding strategy that is distinct from tasks such as continuous spatial alternation, which require the hippocampal network to orthogonalize highly similar trajectories to reduce interference between trials. In addition to differentiating trajectories and, to a lesser extent, defining successive locations occupied while traversing the maze, the DNMP task requires the hippocampal network to alternate between encoding in the sample phase and retrieval in the subsequent choice phase, with information of a current trial not relevant to the demands of the next trial. Our results suggest that there are separate populations of neurons in the hippocampus selectively active during encoding and retrieval and that these components of memory processing are prominent in a spatial task that requires hippocampal function.

\section{References}

Anderson MI, Jeffery KJ (2003) Heterogeneous modulation of place cell firing by changes in context. J Neurosci 23:8827-8835.

Bower MR, Euston DR, McNaughton BL (2005) Sequential-contextdependent hippocampal activity is not necessary to learn sequences with repeated elements. J Neurosci 25:1313-1323.

Breese CR, Hampson RE, Deadwyler SA (1989) Hippocampal place cells: stereotypy and plasticity. J Neurosci 9:1097-1111.

Deadwyler SA, Bunn T, Hampson RE (1996) Hippocampal ensemble activity during spatial delayed-nonmatch-to-sample performance in rats. J Neurosci 16:354-372.

Dudchenko PA, Wood ER, Eichenbaum H (2000) Neurotoxic hippocampal lesions have no effect on odor span and little effect on odor recognition memory but produce significant impairments on spatial span, recognition, and alternation. J Neurosci 20:2964-2977.

Ferbinteanu J, Shapiro ML (2003) Prospective and retrospective memory coding in the hippocampus. Neuron 40:1227-1239.

Frank LM, Brown EN, Wilson M (2000) Trajectory encoding in the hippocampus and entorhinal cortex. Neuron 27:169-178.

Fyhn M, Molden S, Hollup S, Moser MB, Moser E (2002) Hippocampal neurons responding to first-time dislocation of a target object. Neuron 35:555-566.

Gothard KM, Skaggs WE, Moore KM, McNaughton BL (1996a) Binding of hippocampal CA1 neural activity to multiple reference frames in a landmark-based navigation task. J Neurosci 16:823-835.

Gothard KM, Skaggs WE, McNaughton BL (1996b) Dynamics of mismatch correction in the hippocampal ensemble code for space: interaction between path integration and environmental cues. J Neurosci 16:8027-8040.

Hampson RE, Heyser CJ, Deadwyler SA (1993) Hippocampal cell firing correlates of delayed-match-to-sample performance in the rat. Behav Neurosci 107:715-739.

Hampson RE, Jarrard LE, Deadwyler SA (1999) Effects of ibotenate hippocampal and extrahippocampal destruction on delayed-match and -nonmatch-to-sample behavior in rats. J Neurosci 19:1492-1507.

Hasselmo ME, Eichenbaum H (2005) Hippocampal mechanisms for the context-dependent retrieval of episodes. Neural Networks 18:1172-1190.

Hollup SA, Molden S, Donnett JG, Moser MB, Moser EI (2001) Accumulation of hippocampal place fields at the goal location in an annular watermaze task. J Neurosci 21:1635-1644.

Kobayashi T, Nishijo H, Fukuda M, Bures J, Ono T (1997) Task-dependent representations in rat hippocampal place neurons. J Neurophysiol 78:597-613.

Lee I, Rao G, Knierim JJ (2004) A double dissociation between hippocampal subfields: differential time course of CA3 and CA1 place cells for processing changed environments. Neuron 42:803-815.

Lee I, Griffin AL, Zilli EA, Eichenbaum H, Hasselmo ME (2006) Gradual translocation of spatial correlates of neuronal firing in the hippocampus toward prospective reward locations. Neuron 51:639-650.

Lenck-Santini PP, Save E, Poucet B (2001a) Evidence for a relationship between place-cell spatial firing and spatial memory performance. Hippocampus 11:377-390.

Lenck-Santini PP, Save E, Poucet B (2001b) Place-cell firing does not depend on the direction of turn in a Y-maze alternation task. Eur J Neurosci 13:1055-1058.

Leutgeb S, Leutgeb JK, Moser MB, Moser EI (2005) Place cells, spatial maps and the population code for memory. Curr Opin Neurobiol 15:738-746.

Mehta MR, Barnes CA, McNaughton BL (1997) Experience-dependent, asymmetric expansion of hippocampal place fields. Proc Natl Acad Sci USA 94:8918-8921.

Mehta MR, Quirk MC, Wilson MA (2000) Experience-dependent asymmetric shape of hippocampal receptive fields. Neuron 25:707-715.

Muller RU, Kubie JL (1987) The effects of changes in the environment on the spatial firing of hippocampal complex-spike cells. J Neurosci 7:1951-1968.

O'Keefe J, Dostrovsky J (1971) The hippocampus as a spatial map. Preliminary evidence from unit activity in the freely-moving rat. Brain Res 34:171-175.

O'Keefe J, Nadel L (1978) The hippocampus as a cognitive map. Oxford: Oxford UP.

Ranck Jr JB (1973) Studies on single neurons in dorsal hippocampal formation and septum in unrestrained rats. I. Behavioral correlates and firing repertoires. Exp Neurol 41:461-531.

Rivard B, Li Y, Lenck-Santini PP, Poucet B, Muller RU (2004) Representation of objects in space by two classes of hippocampal pyramidal cells. J Gen Physiol 124:9-25.

Rosenzweig ES, Redish AD, McNaughton BL, Barnes CA (2003) Hippocampal map realignment and spatial learning. Nat Neurosci 6:609-615.

Shaw C, Aggleton JP (1993) The effects of fornix and medial prefrontal lesions on delayed non-matching-to-sample by rats. Behav Brain Res 54:91-102.

Skaggs WE, McNaughton BL, Wilson MA, Barnes CA (1996) Theta phase precession in hippocampal neuronal populations and the compression of temporal sequences. Hippocampus 6:149-172.

Smith DM, Mizumori SJ (2006) Hippocampal place cells, context, and episodic memory. Hippocampus 16:716-729.

Tanila H, Shapiro ML, Eichenbaum H (1997) Discordance of spatial representation in ensembles of hippocampal place cells. Hippocampus 7:613-623.

Wible CG, Findling RL, Shapiro M, Lang EJ, Crane S, Olton DS (1986) Mnemonic correlates of unit activity in the hippocampus. Brain Res 399:97-110.

Wood ER, Dudchenko PA, Eichenbaum H (1999) The global record of memory in hippocampal neuronal activity. Nature 397:613-616.

Wood ER, Dudchenko PA, Robitsek RJ, Eichenbaum H (2000) Hippocampal neurons encode information about different types of memory episodes occurring in the same location. Neuron 27:623-633. 